\title{
A Blockchain-based Certificable and Redactable E-taxing Protocol
}

\author{
Huimin Niu, Ting Li, Xiugang Gong* \\ School of Computer Science and Technology, Shandong University of Technology, Zibo, Shandong, China \\ *Correspondence Author: gong_xg@sdut.edu.cn
}

\begin{abstract}
Taxation is a primary source for the government revenue, and its complexity of the tax administration creates loopholes that can be exploited by dishonest taxpayers to minimize the tax paid to the government. We utilize the blockchain technology to create a transparent and secure tax system as well as to simplify the process of administering taxes. However, due to the decentralized and untamperable nature of blockchain may have limitations in tax system applications. In this paper, we propose a blockchain-based certificable and redactable scheme for e-tax. We adopt self-certified public keys to authenticate taxpayers and chameleon hash function to delete (merge) the tax information of related users on the blockchain while keeping the block header unchanged.
\end{abstract}

Keywords: E-taxing, Blockchain, Certifiable, Anonymous.

\section{Introduction}

Taxes are a major source of government revenue. As one of the largest sources of national revenue, the tax sector should always be able to adapt to any development that could occur. With the arrival of bitcoin technology[1], blockchain has also received a considerable amount of attention as the underlying application technology for bitcoin, its application scenarios can be used not only in the context of cryptocurrencies, but also for a wide range of applications in various government[2] and business sectors[3]. In addition, blockchain technology can also be applied efficient tax administration[4]. There has been a lot of research on blockchain applications for taxation, Saragih and Setyowati's research[5] aims to explore the benefits of blockchain and the factors affecting blockchain technology in tax administration. Meanwhile, it is also stated in[6][7] that can reduce the risk of tax avoidance, fraud and evasion, speed up transaction completion, increasing transaction auditability, and increasing the effectiveness of supervision.

However, most blockchains have the property of joint bookkeeping by multiple parties, and when a transaction has been completed, no party can change its transaction records[8]. Although the authenticity of business processes is ensured at the root, with the exponential growth of the equipment performance, some security problems begin to show in the blockchain. Powerful attacks, such as $51 \%$ attacks, will cause wrong data being recorded on the blockchain and lead to catastrophic consequences[9]. An unchangeable record is not useful for the applications that area used for blockchain. The non tamperability of blockchain could limit the wide uses in financial sector for the services, such as changing and shredding data, an oversight, an attack, or coding and transaction mistakes, which can be undone through redaction[10]. While this is an essential feature of most blockchain scenarios, it is still often desirable at times it may be even legally required to allow for breaking this immutability in a controlled way. For example, the upcoming general data protection regulations(GDPR) of EU imposes the Right to be Forgotten as a key Data Subject Right. So, the redactable blockchain were invented to allow the ledger to mutate in a controlled manner[11]. The application of blockchain in e-tax system is relatively new and not widely accepted. Although many scholars have discussed the advantages of combining blockchain with e-tax system, they have not proposed its specific and relevant scheme construction.

Contributions: We constructed a specific blockchain-based protocol in the e-tax system for solving the current challenges it faces. The main contributions of this paper can be summarized as follows.

- We proposed an identify self-certified scheme for e-tax system. Taxpayer's private key can be jointly computed by the taxpayer and the certificate authority. The certificate is embedded in the selfcertified public key. Verifying validity of the signature and the public key can be concurrently accomplished in a logically single step. Therefore, it can further realize the privacy protection, and reduce the computation and storage overhead.

- We further provide an efficient blockchain redacting method by using chameleon hash. Tax authority can modify illegal information on the blockchain at any time, or merge huge financial data. Tax authority can rewrite the contents of the block so that overwriting the improper information in the blockchain.

Organization. In section 2, we review the background knowledge and basic definition. Then, we introduce the e-tax system model and security requirements in section 3 . A detailed description of proposed e-tax protocol in section 4. Finally, the security analysis of our scheme is given in section 5 .

\section{Preliminaries}

In the section, we illustrate background knowledge used in the paper.

\subsection{Self-certified Public Key}

In 1991, Girault[12] first proposed a self-certified public key system to resolve the problem of public key verification. Self- 
certified public key is intermediary between certificatebased[13] and identity-based[14]. It are neither certificatebased, since there is no separate certificate, nor identity-based, since the public key is not restricted to identity. They contribute to reduce the amount of storage and computations while secret keys are still chosen by the user himself and remain unknown to the authority.

\subsection{Chameleon Hash Function}

A chameleon hash is a cryptographic hash function that contains a trapdoor, it is anti-collision for outside, and it uses a trapdoor information to generate the collisions efficiency.

Definition(Chameleon-Hash) The chameleon hash function[15] consists of four algorithm $\mathrm{CH}=(\mathrm{HGen}, \mathrm{Hash}$, $\mathrm{HVer}, \mathrm{HCol}$ ). The algorithm details are follows.

$\operatorname{HGen}\left(1^{\lambda}\right) \rightarrow(h k, t k):$ The key generation algorithm inputs the security parameter $\lambda$, and outputs the public hash key $h k$, and a secret trapdoor key $t k$.

$\operatorname{Hash}(h k, m, r) \rightarrow h$ : The chameleon hash algorithm takes the hash key $h k$ a message $m$ and random number $r$ used to generate the hash value as input, and output as $h=\operatorname{Hash}(h k, m, r)$.

$H \operatorname{Ver}(h k,(m, h, r)) \rightarrow d:$ The verification algorithm takes the hash key $h k$, a message $m$, and $(h, r)$ as input, and outputs $d \in\{0,1\}$. It returns $d=1$ if and only if $\operatorname{Hash}(h k, m, r)=h$. Otherwise, $d$ equals 0 .

$H C \operatorname{Col}\left(t k,(m, h, r), m^{\prime}\right) \rightarrow r^{\prime}$ : The collision algorithm inputs the new message $m^{\prime}$, the random number $r$, the private key $t k$ and the origin message $m$, we can output a new $r^{\prime}$ satisfying $\operatorname{Hash}(m, r, h k)=\operatorname{Hash}\left(m^{\prime}, r^{\prime}, h k\right)$.

\section{System Model and Security Requirements}

In the section, we introduce the system model and components, security requirements of our proposed scheme.

\subsection{System Model and Components}

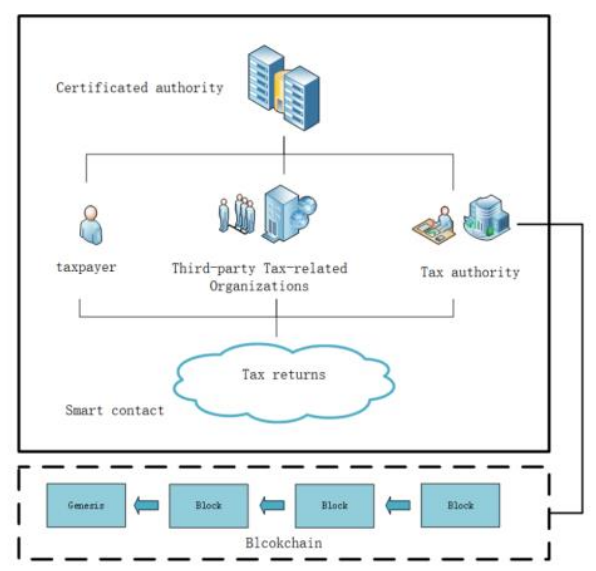

Figure 1: The framework of the blockchain-based e-tax system
As shown in Figures 1, five entities are involved in our system, namely, the certificate authority $(C A)$, the third-party taxrelated institutions $(T E)$, the tax authority $(T A)$, the taxpayer( $T U)$ and smart contract $(S C)$.

Certificate authority is in charge of the initialization of system parameters and the registration of all third-party institutions, such as financial institutions and medical institutions, etc, the tax authority, and taxpayers. Meanwhile, it can also reveal the real identity of a malicious taxpayer. We assume that certificate authority is fully trusted and cannot be compromised. In our scheme, tax authority deploys smart contracts and audits the tax return. Third-party tax-related institutions publishes the tax payment information of the corresponding taxpayer to smart contracts.

\subsection{Security Requirement}

In a blockchain-based e-tax system, we aim to achieve the following goals.

Anonymity: anonymity in our solution refers to the anonymity of the user's identity. Usually, anonymity is only for legitimate taxpayers, whose data security is crucial.

Unforgeability: the unforgerability of tax returns is also the guarantee of taxpayers' rights, so the unforgerability of tax returns is realized to ensure the uniqueness and authenticity, so that each process can be verified.

Accountability: the accountability should be done for an illegal taxpayer. When a tax return is falsified or a taxpayer tries to evade taxes, authority can track the tax return of the malicious taxpayer and reveal his/her true identity.

Efficient: e-tax system must be efficient. It means not only should tracing (anonymity revocation) be performed efficiently, but the adding burden to the basic system should be minimal for all involved parts - certificate authority, tax authority, third-party institutions, taxpayer. In particular, certificate authority must be involved only when revocation is required and remain off-line otherwise.

\section{Construction}

Here we give a detailed description about our proposed system, which have six phases: 1) Initialization phase. 2) Register phase. 3) Declaration phase. 4) Audit phase. 5) Transactions block generation phase. 6) Redacting phase.

1) Initialization phase. The initialization phase can be divided into two phase. One part is the certificate authority initialization, the other part is tax authority and third-party institutions, which generates the its secret key and public key. The detailed steps are described follows.

- The $C A$ inputs a security parameter $\lambda \in Z^{*}$ and returns system parameters. Given a group $G_{p}$ over a finite field $F_{p}$ of prime order $p$ where $P$ is a base point generator. The $C A$ chooses a secret value $S_{C A} \in Z_{p}^{*}$ as his private key and computes his public key $P_{C A}=S_{C A} \cdot P$. The secure hash 
function $H:\{0,1\}^{*} \rightarrow G_{p}$

- The $T A$ inputs random value $S_{T A} \in Z_{p}^{*}$ as the secret trapdoor key, and computes the public hash key $P_{T A}=S_{T A} \cdot P$.

- Similarly, $T E_{j}$ randomly chooses its own private key $S_{T E_{j}} \in Z_{p}^{*}$ and calculates their public key $P_{T E_{j}}=S_{T E_{j}} \cdot P$

- At the end of setup phase, each party keeps their own private keys, and $C A$ preloaded with the public parameters $\left\{G_{p}, F_{p}, E / F_{p}, P, P_{C A}, P_{T A}, P_{T E_{j}}, H(\cdot)\right\}$.

2) Register phase: A user needs to register to become a legitimate taxpayer. Get a tax identification number by registering with $C A$, which is unique.

- A user's real identity is $R I D_{i}$, every user chooses a random secret $x_{i} \in Z_{p}^{*}$ then computes $X_{i}=H\left(R I D_{i} \| x_{i}\right) \cdot P$. Thereafter, the user sends a message $\left\langle R I D_{i}, X_{i}>\right.$ to $C A$ through a secure channel (SSL) that sets up an encrypted connection between the user and the server.

$$
\text { user }_{i} \rightarrow C A:<R I D_{i}, X_{i}>
$$

- $C A$ maintains an initially-empty registry list. The $C A$ receives the message tuple from user, and chooses a random secret $k_{i} \in Z_{p}^{*}$ to compute user's partial private key $\bar{S}_{i}$ along with a witness $W_{i}$ to verify his legitimacy as follows.

$$
\begin{gathered}
e_{i}=H\left(R I D_{i} \| k_{i}\right) \cdot S_{C A} \\
T I D_{i}=X_{i} \oplus e_{i} \\
W i=X_{i} \oplus k_{i} \cdot P \\
\bar{S}_{i}=H\left(T I D_{i} \| W_{i}\right) \cdot S_{C A} \oplus k_{i}
\end{gathered}
$$

After that, $C A$ sends the value $\left\langle R I D_{i}, T I D_{i}, W_{i}, \bar{S}_{i}\right\rangle$ in a secure SSL channel to the user. $T I D_{i}$ is taxpayer's tax identification number.

$$
C A \rightarrow \text { user }_{i}:<R I D_{i}, T I D_{i}, W_{i}, \bar{S}_{i}>
$$

- After receiving the message, user computes a pair of his own private key $S_{i}$ and extracts public key $P_{i}$.

$$
\begin{gathered}
S_{i}=\bar{S}_{i} \oplus H\left(R I D_{i} \| x_{i}\right) \\
P_{i}=S_{i} \cdot P
\end{gathered}
$$

Further, the keys can be verified as $S_{i} \cdot P=\left[H\left(T I D_{i} \| W_{i}\right)\right]$, if this function holds correct then user accepts his/her private key else rejects. The correctness of the public key derivation follows.

3) Declaration phase: Taxpayer submits tax returns to $T A$ within deadline online using a web application. $S C$ allow taxpayer to check tax information and fill out the tax return. The $T U_{i}$ generates a time-stamp $T_{i}$ and tax return $m_{i} \in\{0,1\}^{*}$, which we denote by $m_{i}$ contains: public key $P_{T E}$ of third- party tax related institutions, $T U_{i}$ 's tax identification number $T I D_{i}, T A$ 's public key $P_{T A}$, etc. In this phase, the $T U_{i}$ computes signature, the sends a computed list of parameters encrypted using a symmetric encryption algorithm (AES) to establish a secure connection with $T A$.

- Firstly, $T U_{i}$ chooses a random value $\alpha_{i} \in Z_{p}^{*}$, and computes signature. The steps are shown as follows.

$$
\begin{gathered}
\alpha_{i} P=R=\left(R_{x}, R_{y}\right) \\
\sigma_{1}=R_{x} \\
\sigma_{2}=\alpha_{i}+H\left(m_{i}\left\|\sigma_{1}\right\| P_{T E_{j}}\right) \cdot S_{i}
\end{gathered}
$$

- Second, $T U_{i}$ generates a symmetric session key and encrypts the signature. The specific steps are as follows.

$$
W_{1}=T I D_{i} \oplus W_{i}
$$

$T U_{i}$ chooses a random value $d_{i} \in Z_{p}^{*}$ and computes symmetric session key key $_{i}$.

$$
k e y_{i}=H\left(S_{i} \cdot P_{T A}\left\|d_{i}\right\| T_{i}\right)=\left(\text { key }_{i x}, \mathrm{key}_{i y}\right)
$$

where $k e y_{a x}$ is an $x$-coordinate derived using ECC-point on the curve to encrypt the message and signature.

$$
M_{1}=H\left(d_{i}\left\|W_{i}\right\| k e y_{i x}\right)
$$

Thereafter sends the message tuple $\left\{\left(\sigma_{1}, \sigma_{2}\right), m_{i}, T I D_{i}, M_{1}\right.$, $\left.W_{i}, d_{i}, T_{i}, P_{T E_{j}}\right\}$ encrypted with $k e y_{a x}$.

$$
\begin{gathered}
T U_{i} \rightarrow T A:<P_{i}, d_{i}, T_{i}, T I D_{i}, \\
E n_{\text {key }_{a x}}\left\{\left(\sigma_{1}, \sigma_{2}\right), m_{i}, T I D_{i}, M_{1}, W_{i}, d_{i}, T_{i}, P_{T E_{j}}\right\}>
\end{gathered}
$$

4) Audit phase: Upon receiving the message from taxpayer, $T A$ verifies whether the timestamp $T_{i}$ is within the allowed range compared to current time. If not, $T A$ rejects the request, otherwise, $T A$ decrypts message get the message tuple, and verifies the signature.

- Firstly, $T A$ computes the symmetric key for decrypting the received message as following.

$$
k e y_{i}^{\prime}=H\left(S_{T A} \cdot P_{i}\left\|d_{i}\right\| T_{i}\right)
$$

Once $k e y_{i}^{\prime}$ is computed then its $x$ and $y$ coordinates are identified. Using the $x$-coordinates $k e y_{i x}^{\prime}$, the message is decrypted to obtain $\left\{\left(\sigma_{1}, \sigma_{2}\right), m_{i}, M_{1}, P_{T E_{j}}\right\}$, then performs a mutual verify process using the received parameters.

- It is worth noting that in the process of verifying the signature, $T U_{i}$ need not provide any additional certificate to prove the authenticity of $P_{i}$, the public key can be completed logically at the same time as signature verification. $T A$ computes as following.

$$
\begin{gathered}
W_{1}^{\prime}=T I D_{i} \oplus W_{i} \\
M_{i}^{\prime}=H\left(d i\left\|W_{1}^{\prime}\right\| k e y_{i x}^{\prime}\right) \\
P_{i}^{\prime}=H\left(T I D_{i} \oplus W_{i}\right) \cdot P_{C A} \oplus W_{i}
\end{gathered}
$$




$$
\begin{gathered}
\alpha_{i} \cdot P \stackrel{?}{=} \sigma_{2} \cdot P-H\left(m_{i}\left\|\sigma_{1}\right\| P_{T E_{j}}\right) \cdot P_{i} \\
R_{x} \stackrel{?}{=} \sigma_{1}
\end{gathered}
$$

5) Transactions block generation phase: In this phase, creating a block that contains all the taxpayers' identities, public key, and signature of the tax return.

- First, for each taxpayer $T I D_{i}$, set $\lambda_{i}=\left\{T I D_{i}, P_{i}\right.$, $\left.\operatorname{Sig}_{S_{T U_{i}}}\left\{m_{i}\right\}\right\}, \omega_{j}=\left\{\lambda_{1 j}, \lambda_{2 j}, \ldots, \lambda_{i j}\right\} . T E$ generates signatures for all user's tax returns, i.e. $\operatorname{Sig}_{S_{T E_{j}}}\left\{H\left(\omega_{j}\right)\right\}$, set $M_{j}=\left\{T E_{j}, \omega_{j}, \operatorname{Sig}_{S_{T E_{j}}}\left\{H\left(\omega_{j}\right)\right\}\right\}$.

- Second, $T A$ computes the message $M$ hash value.

a. Select random value $r_{1}$, where $r_{1} \in Z_{p}^{*}$;

b. Compute chameleon hash value $h=H\left(M_{j}, r_{1}\right)=$ $f\left(M_{i}\right) \cdot P_{T A}+r_{1} \cdot P$. TA adds the taxpayer's transaction information to blockchain.

c. Tax return $t r_{j}=\left\{M_{j}, h\right\}$.

6) Redacting phase: In general, $T A$ needs to maintain and periodically update a block. In our scheme, we utilize chameleon hash to modify block information instead of the old one. If a user can be considered as an illegal network user. In order to delete the tax information on the blockchain, the $T A$ generates a new transaction that does not contain illegal user's tax information on the blockchain. After the new message is verify by $T A$, it replaces the original message. The specific redaction blockchian steps are as follows.

- If $T A$ needs to revoke user $T U_{i}$, whose information is contained in $t r_{j}, T A$ first generates a new message $M_{j}^{\prime}=\left\{T E_{j}, \omega_{j}^{\prime}, \operatorname{Sig}_{S_{T E_{j}}}\left\{H\left(\omega_{j}^{\prime}\right)\right\}\right\}$, where $\lambda_{k j}=\left\{T I D_{k}, P_{k}\right.$, $\left.\operatorname{Sig}_{S_{T U_{k}}}\left\{m_{k}\right\}\right\}, \omega_{j}^{\prime}=\left\{\lambda_{1 j}, \lambda_{2 j}, \ldots, \lambda_{(i-1) j}\right\} . M_{j}^{\prime}$ is the same as $M_{j}$ except that $M_{j}^{\prime}$ does not contain the tax return information of $U_{i}$.

- $T A$ generates chameleon hash collision with $M_{j}^{\prime}$ and its private trapdoor key.

a.Compute updated $r_{1}^{\prime}=r_{1}+S_{T A} \cdot\left[f\left(M_{j}\right)-f\left(M_{j}^{\prime}\right)\right]$

b. Updated tax return $\operatorname{tr}_{j}^{\prime}=\left\{M_{j}^{\prime}, h\right\}$.

\section{Security Analysis}

In this section, we analyze the security of our proposed scheme according to the design scheme described in the previous section.

Theorem 1 The proposed protocol can preserver taxpayer's anonymity.
Proof: In our scheme, the taxpayer $T U_{i}$ sends the message $<P_{i}, d_{i}, T_{i}, T I D_{i}, \operatorname{En}_{k e y_{a x}}\left\{\left(\sigma_{1}, \sigma_{2}\right), m_{i}, T I D_{i}, M_{1}, W_{i}, d_{i}, T_{i}, P_{T E_{j}}\right\}>$ to the $T A$ where the taxpayer's identity is encrypted using a symmetric-key operation over an insecure channel. In the process of tax authority and taxpayer session, even if a adversary intercepts the message, he cannot obtain the real identity of taxpayer, because the tax return includes a identity $T I D_{i}$ instead of $R I D_{i}$, i.e. $T I D_{i}=X_{i} \oplus e_{i}$, where $e_{i}$ is computed by $C A$ using a one-way hash function and two random secrets $k_{i}$ and $S_{C A}$. Also, $X_{i}$ is is computed by the $T U_{i}$ using a random $x_{i}$ and a one-way hash function. Therefore, it is difficult for an adversary to compute a real identity without the knowledge of these random secrets. Thus our scheme preserves user anonymity.

Theorem 2 The proposed protocol can preserve trace attack.

proof: In our scheme, during the declaration phase, each message is encrypted and sent along with his/her tax identification number, not their real identity. Therefore, it is infeasible for an attacker to intercept messages and track their private information related to their current salary, lifestyle habits, etc. Therefore, our scheme protects taxpayer's privacy by achieving untraceability.

Theorem 3 The proposed protocol can preserve replay attack.

proof: $T U_{i}$ and $T A$ uses timestamps and random numbers as interrogation responses during the transmission of messages. We consider the following attack scenarios. Suppose an adversary intercepts the message in declaration phase, and tries to replay the message $<P_{i}, d_{i}, T_{i}^{*}, T I D_{i}, E n_{k e y_{a x}}\left\{\left(\sigma_{1}, \sigma_{2}\right)\right.$, $\left.m_{i}, T I D_{i}, M_{1}, W_{i}, d_{i}, T_{i}, P_{T E_{j}}\right\}>$ Then the $T A$ accepts the message and computes $k e y_{i}^{*}$. Here, the original $k e y_{i}$ is infeasible to compute due to difficulty in solving computational Diffie-Hellman problem. The adversary cannot compute the correct key based on different timestamps $T_{i} \neq T_{i}^{*}$ and unknown $T U_{i}$ 's private key $S_{i}$. Thus our proposed protocol preserves replay attack.

Theorem 4 The proposed protocol can preserve a knownkey attack.

proof: In our scheme, our protocol is said to be known-key secure if the previous declarationkey disclosure will not affect the future declaration. Suppose the current declaration-key key $_{i}^{\prime}=H\left(S_{T A} \cdot P_{i}\left\|d_{i}\right\| T_{i}\right)$ is compromised, which does not reveal any information about other declaration keys. The declaration key is hashed with one-way hash function, and solving the key problem requires not only knowing the random number $d_{i}$ and the private key $S_{i}$, but also solving the $E C D L P$ problem in polynomial-time, which is apparently impossible. Therefore, the proposed protocol can preserve a known-key attack.

\section{Conclusion}

In this paper, we propose an efficient certificable and redactable scheme using self-certified public key and 
chameleon hash function. Selfcertified public key technology not only reduces the leakage of privacy, i.e. taxpayers' identifying information is completely controlled and managed by the owner, but also implements identity verification at the same time as the tax authority verifies the signature phase. Moreover, by applying chameleon hash function in our scheme, tax authority can actively maintain and redact the blockchain. Finally, after an extended security analysis, we proved that our scheme is suitable for e-tax.

\section{References}

[1] Nakamoto S, Bitcoin A. A peer-to-peer electronic cash system[J]. Bitcoin. URL: https://bitcoin. org/bitcoin.pdf, 2008, 4.

[2] Ølnes S, Ubacht J, Janssen M. Blockchain in government: benefits and implications of distributed ledger technology for information sharing[J]. Government Information Quarterly, 2017, 34(3): 355-364.

[3] Moşteanu N R, Faccia A. Digital systems and new challenges of financial management-FinTech, XBRL, blockchain and cryptocurrencies[J]. Quality-Access to Success Journal, 2020, 21(174): 159-166.

[4] Setyowati M S, Utami N D, Saragih A H, et al. Blockchain technology application for value-added tax systems[J]. Journal of Open Innovation: Technology, Market, and Complexity, 2020, 6(4): 156.

[5] Saragih S M F, Milla S S. E-Readiness of blockchain technology in modernization of tax administration in Indonesia[J]. 2019.

[6] Faccia A, Mosteanu N R. Tax evasion information system and blockchain[J]. Journal of Information Systems \& Operations Management, 2019, 13(1).

[7] Ali S. Revolusi industri 4.0 dan dampaknya terhadap pendidikan akuntansi di Indonesia[J]. BPFE Yogyakarta, 2019.

[8] Choi B G, Jeong E S, Kim S W. Multiple security certification system between blockchain based terminal and internet of things device: Implication for open innovation[J]. Journal of Open Innovation: Technology, Market, and Complexity, 2019, 5(4): 87.

[9] Ali M, Nelson J, Shea R, et al. Blockstack: A global naming and storage system secured by blockchains[C]//2016 USENIX annual technical conference, 2016: 181-194.

[10] Rajasekhar K, Yalavarthy S H, Mullapudi S, et al. Redactable blockchain and it's implementation in bitcoin[J]. International Journal of Engineering \& Technology, 2018, 7(1.1): 401-405.

[11] Dousti M S, Küpçü A. Tri-op redactable blockchains with block modification, removal, and insertion[J]. Cryptology ePrint Archive, 2021.

[12] Girault M. Self-certified public keys[C]//Workshop on the Theory and Application of Cryptographic Techniques. Springer, Berlin, Heidelberg, 1991: 490497.

[13] Recomendation C D. The Directory Authentication Framework[J]. 1987.

[14] Guillou L C, Quisquater J J. A practical zero-knowledge protocol fitted to security microprocessor minimizing both transmission and memory[C]//Workshop on the theory and application of of cryptographic techniques. Springer, Berlin, Heidelberg, 1988: 123-128.

[15] Ateniese G, Magri B, Venturi D, et al. Redactable blockchain-or-rewriting history in bitcoin and friends[C]//2017 IEEE European symposium on security and privacy (EuroS\&P). IEEE, 2017: 111-126. 